\title{
Intrapancreatic accessory spleen: Evaluation with CT and MRI
}

\author{
QIANJIANG DING ${ }^{1 *}$, ZHIHAO REN $^{1 *}$, JIANHUA WANG $^{1}$, XIAOLONG MA $^{2}$, JIAN ZHANG $^{3}$, \\ GAOFENG SUN ${ }^{3}$, CHANGJING ZUO ${ }^{3}, \mathrm{HAO} \mathrm{GU}^{4}$ and HUI JIANG ${ }^{5}$ \\ ${ }^{1}$ Department of Radiology, The Affiliated Hospital of Medical School, Ningbo University, Ningbo, Zhejiang 315020; \\ Departments of ${ }^{2}$ Radiology and ${ }^{3}$ Nuclear Medicine, Changhai Hospital, Second Military Medical University, \\ Shanghai 200433; ${ }^{4}$ Department of Pathology, Ningbo Second Hospital, Ningbo, Zhejiang 315010; \\ ${ }^{5}$ Department of Pathology, Changhai Hospital, Second Military Medical University, Shanghai 200433, P.R. China
}

Received March 7, 2017; Accepted March 23, 2018

DOI: $10.3892 / \mathrm{etm} .2018 .6613$

\begin{abstract}
The aim of the present study was to evaluate the characteristics of computed tomography (CT) and magnetic resonance imaging (MRI), particularly diffusion-weighted imaging (DWI), in the imaging of intrapancreatic accessory spleen (IPAS). The clinical and pathological records of 9 patients, including 8 patients with IPAS and 1 patient with splenosis, were reviewed. The patients had undergone plain and triple-phase enhanced CT scanning ( $\mathrm{n}=9$ ) and MRI scanning $(\mathrm{n}=8)$. The lesions of the 8 IPAS patients were located in the pancreatic tail, and were round $(n=3)$, oval $(n=4)$ or triangular $(\mathrm{n}=1)$ in shape. The $\mathrm{CT}$ and/or MRI densities, signal intensities and dynamic enhanced patterns of the lesions were similar to those of the orthotopic spleen. In DWI images $(n=5)$, the IPAS regions presented high signal intensity (SI), and no significant difference in the apparent diffusion coefficient determined using a b-value of $600 \mathrm{sec} / \mathrm{mm}^{2}$ was identified between the IPAS and orthotopic spleen $(\mathrm{P}>0.05)$. One patient with splenosis complicated with cirrhosis had a nodule located in the pancreatic tail with an unenhanced CT value of $65 \mathrm{HU}$. In MRI examination, with the exception of the dynamic enhancement pattern, the T1-weighted, T2-weighted and DWI signals of splenosis were inconsistent with those of the normal spleen. In conclusion, in pre-contrast and post-contrast-enhanced CT and MRI images, IPAS exhibits similar characteristics to the orthotopic spleen. CT and MRI used in combination with DWI are important in the diagnosis of IPAS.
\end{abstract}

Correspondence to: Dr Jianhua Wang, Department of Radiology, The Affiliated Hospital of Medical School, Ningbo University, 247 Renmin Road, Ningbo, Zhejiang 315020, P.R. China

E-mail: woxingw@sina.com

*Contributed equally

Key words: computed tomography, magnetic resonance imaging, diffusion-weighted imaging, intrapancreatic accessory spleen

\section{Introduction}

Intrapancreatic splenic tissue, including splenosis and intrapancreatic accessory spleen (IPAS), often appears in the pancreatic tail $(1,2)$. Splenosis is usually caused by the autotransplantation of splenic tissue, which frequently occurs following splenectomy or spleen trauma; accessory spleens are the congenital foci of healthy splenic tissue that have separated from the main body of the spleen $(3,4)$. However, intrapancreatic splenic tissue is challenging to identify using medical imaging (5). Intrapancreatic splenic tissue is often misdiagnosed as various pancreatic tumors, including islet cell tumor, solid pseudopapillarytumor, hypervascular metastasis and ductal adenocarcinoma (5). Accurate preoperative diagnosis is of great importance to avoid unnecessary surgery or biopsy.

Several studies have provided certain analyses on the imaging features of intrapancreatic splenic tissue and their correlation with the pathological findings (6-16). The CT scans in these studies demonstrated a well-circumscribed, enhancing mass in the tail of the pancreas that revealed similar enhancement patterns as the spleen. The specimens contained well-demarcated dark-red nodules surrounded by pancreatic tissue. The nodular lesions were elastic and soft, and composed of lymphoid follicles and splenic pulp. Although IPASs have been reported, the majority of the studies were case reports, and lacked comprehensive conclusions about imaging features. To the best of our knowledge, the performance of diffusion-weighted magnetic resonance imaging (DWI) in the detection of IPAS has not yet been reported. In the present study, the computed tomography (CT) and magnetic resonance imaging (MRI) examination results of 9 cases of intrapancreatic spleen were investigated, with a particular focus on the features of DWI.

\section{Materials and methods}

Patients. The present retrospective review of patient data was approved by the Ethics Committee of Ningbo University Hospital (Ningbo, China). From July 2010 to July 2015, the clinical and pathological records, as well as the imaging examinations of patients at the Affiliated Hospital of the 
Medical School of Ningbo University (Ningbo, China) and the Changhai Hospital of the Second Military Medical University (Shanghai, China) were reviewed. In total, 9 patients were diagnosed with intrapancreatic splenic tissue, including 8 cases of IPAS and 1 case of intrapancreaticsplenosis. The types of clinicoradiological examinations conducted for each patient are presented in Table I.

The 8 patients diagnosed with IPAS were aged between 44 and 65 years (mean age, $56.4 \pm 6.9$ years), included 4 males and 4 females, and had no trauma to the spleen or history of surgery. The serum cancer antigen 19-9 data for the patients were all within the normal range $(0-35 \mu \mathrm{g} / \mathrm{ml})$. The levels of blood glucose and pancreatic enzymes were also normal. Among the 8 patients with IPAS, 2 patients were suspected of having a pancreatic tail tumor when examined by ultrasound imaging due to upper abdominal pain. Another 2 patients had chronic liver dysfunction, one of whom was suspected of having hepatocellular carcinoma as their serum a-fetoprotein level was elevated $(>1,000 \mu \mathrm{g} / \mathrm{l})$ compared with the normal range $(0-20 \mu \mathrm{g} / \mathrm{l})$. The remaining 4 patients did not have any abdominal discomfort or complaints, and were diagnosed with pancreatic tail tumors by abdominal sonography during health examinations. Among the 8 patients with IPAS, 3 patients were confirmed to have IPAS by the examination of a surgical specimen obtained by distal pancreatectomy and splenectomy. These surgeries were performed due to the possibility of neoplastic lesions, although accessory spleen was also radiologically suspected. The remaining 5 patients were suspected of having IPAS on the basis of imaging characteristics and clinical follow-up for 17-38 months (Table I).

The single case of intrapancreaticsplenosis was observed in the pancreatic tail. The patient (male, 55 years old) had a history of splenectomy due to cirrhosis with hypersplenism 23 months previously. No abnormalities of the pancreatic tail were detected by preoperative imaging and intraoperative exploration. No notable changes of the pancreatic tail lesions were identified on the follow-up MRI results at 15 months (Table I).

Pathological analysis. In the 3 cases where PAS was confirmed by pathological analysis, histological sections of the IPAS were prepared by fixation with $10 \%$ formaldehyde at $25^{\circ} \mathrm{C}$ for $24 \mathrm{~h}$ and embedding in paraffin, and then were stained with hematoxylin and eosin. Then, the $3-\mu \mathrm{m}$-thick sections were treated with $1 \%$ periodic acid solution for $10 \mathrm{~min}$ at room temperature, washed with PBS for 5 min, stained with Schiff solution for $10 \mathrm{~min}$ and eosin for $2 \mathrm{~min}$ at $25^{\circ} \mathrm{C}$, and washed with PBS for $10 \mathrm{~min}$. When the section turned red, they were stained with hematoxylin at $60^{\circ} \mathrm{C}$ for $1 \mathrm{~min}$. The diagnosis was made by one experienced pathologist under a light microscope at a magnification of $\mathrm{x} 100$.

Imaging techniques. All 9 patients underwent CT scanning using a Sensation Cardiac $64 \mathrm{CT}$ scanner (Siemens Healthineers, Erlangen, Germany; $\mathrm{n}=8$ ) or a Brilliance $16 \mathrm{CT}$ scanner (Philips Healthcare, Amsterdam, The Netherlands; $\mathrm{n}=1$ ). The sequences consisted of pre-contrast, arterial, portal venous and delayed phases. Following the injection of $100 \mathrm{ml}$ nonionic contrast material (Ultravist 350; Schering AG, Berlin, Germany) at a rate of $2.5-3 \mathrm{ml} / \mathrm{sec}$, triple-phase CT scans were acquired at 25, 60-70 and $120 \mathrm{sec}$ from the initiation of the intravenous injection of the contrast material. The scanning parameters for the two CT scanners were identical, as follows: Slice thickness, $5 \mathrm{~mm}$ without gap; tube voltage, $120 \mathrm{kV}$; tube current, $200 \mathrm{~mA}$; matrix 512x512.

A phased-array abdominal coil was applied to 8 patients with a 1.5-T Avanto MRI scanner (Siemens Healthineers) 3 days after the CT examination. For these patients, T1-weighted imaging (T1WI), T2-weighted imaging (T2WI), triple-phase enhanced scanning on the axial plane and true fast imaging with steady state precession sequences in coronal images were conducted. DWI scanning was also performed for 6 of the 8 patients.

The turbo spin-echo sequence was used for T2WI with the following parameters: Repetition time (TR)/echo time (TE), 3,000-4,500 msec/70-90 msec; flip angle, $150^{\circ}$; matrix, 256x192; and section thickness, $5 \mathrm{~mm}$ without intersection gap. The volumetric interpolated breath-hold sequence was used for T1WI during the pre- and post-contrast phases with the following parameters: TR/TE, $3.49 \mathrm{msec} / 1.02 \mathrm{msec}$; reconstruction thickness, $5 \mathrm{~mm}$; and matrix, 256x128-192. The echo planar imaging sequence was used for DWI with the following parameters: TR/TE, 2,100 msec/127 msec; section thickness, $5 \mathrm{~mm}$; matrix, 192x192; number of excitations, 4-6; and b-values, 0, 100 and $600 \mathrm{sec} / \mathrm{mm}^{2}$. Triple-phase enhanced MRI scans were acquired at 25, 60-70 and $120 \mathrm{sec}$ from the initiation of the intravenous injection of contrast material. A power injector was used to administer gadolinium chelate (Magnevist; Bayer AG, Leverkusen, Germany) at a dose of $0.1 \mathrm{mmol} / \mathrm{kg}$ using an injection rate of $2.5-3 \mathrm{ml} / \mathrm{sec}$.

Image analysis. All images were retrospectively evaluated in consensus by two experienced abdominal radiologists who were unaware the histopathological diagnosis. The following morphological features of the tumors were recorded: Location, tumor size (the maximum cross-sectional diameter of a tumor was defined as the longest measured diameter in the axial scan images; and the minimum as the shortest measured diameter), lesion contour, border definition, density and signal intensity (SI) on CT and MRI images compared with normal pancreas tissue, apparent diffusion coefficient (ADC) values on DWI, and the pattern of contrast enhancement. For the ADC values, the region of interest (ROI) was circled. The ROI encompassed the maximum diameter of the pancreas or lesion, and the area and shape of ROI were as consistent as possible, avoiding the vascular and external structure of the pancreas.

Statistical analysis. The ADC values of the IPAS, orthotopic spleen and pancreatic tissue from the same patient were compared using one-way analysis of variance followed by least significant difference tests. SPSS 18.0 software (SPSS, Inc., Chicago, IL, USA) was used to conduct the analysis and $\mathrm{P}<0.05$ was considered to indicate a statistically significant difference.

\section{Results}

MRI and CT image features of IPAS

Lesion location, size and contour. Among the 8 patients with IPAS, only one lesion was identified at the tail of the 


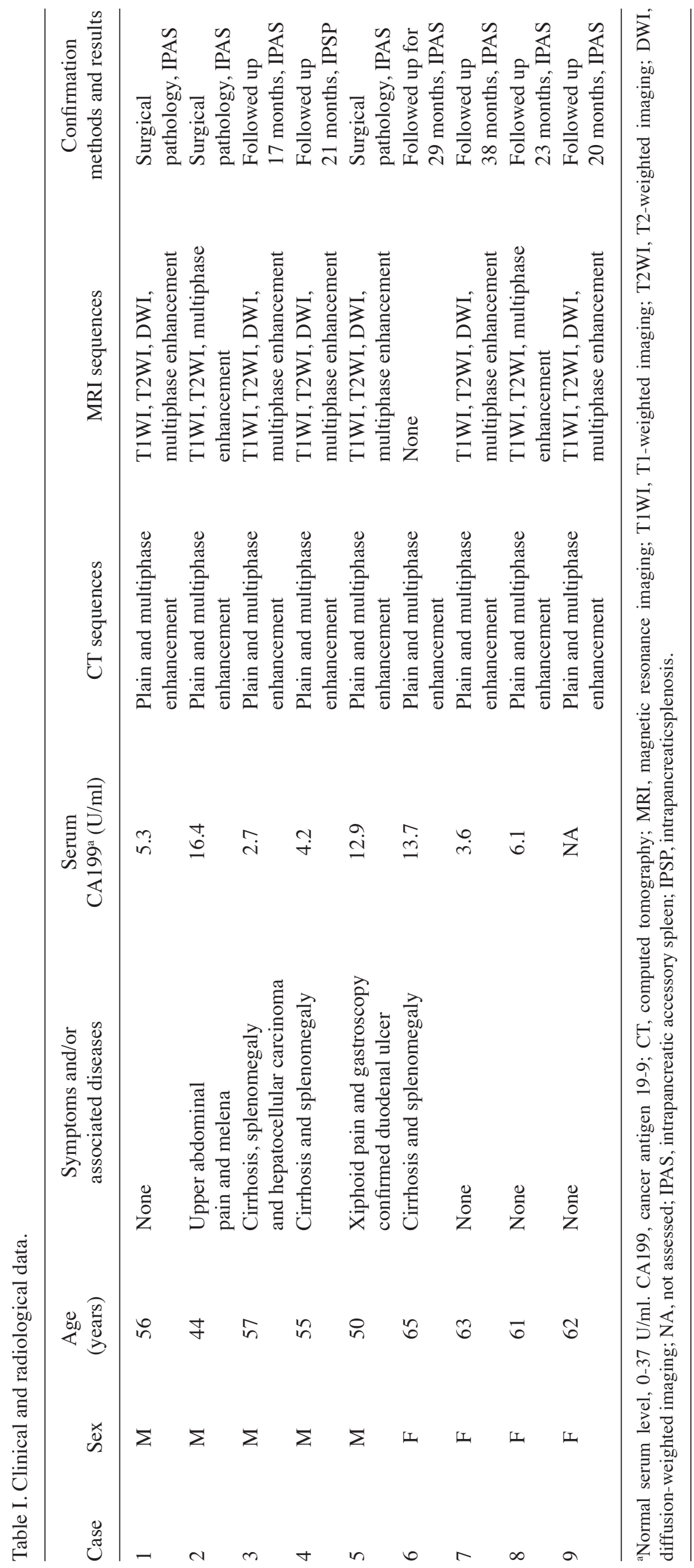



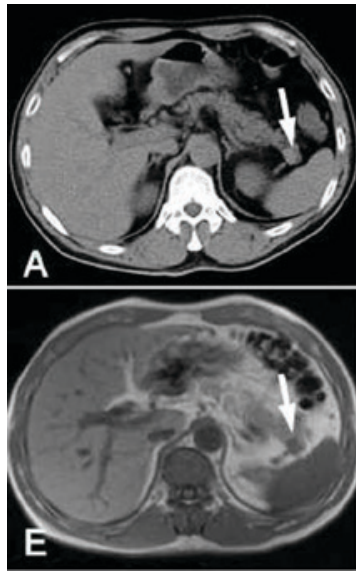
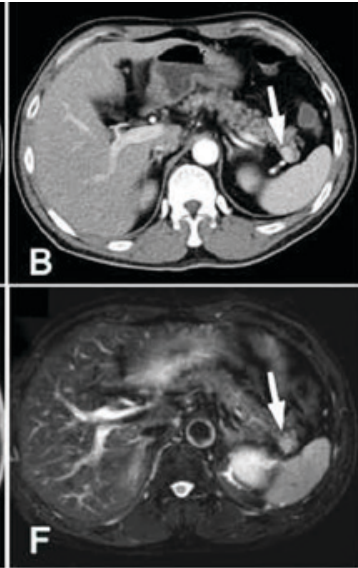


Figure 1. Case 5. CT and MRI images of a 50-year-old male with IPAS. (A) In the pre-contrast CT image, slight hyper-attenuation of the lesion was visible in the pancreatic tail compared with the pancreas (arrow). Contrast-enhanced axial CT images were obtained in the (B) arterial phase, (C) portal venous phase and (D) delayed phase. High attenuation was shown for the IPAS (arrows) compared with the pancreas in all phases, and the attenuation level of the IPAS was comparable with that of the orthotopic spleen. The lesion (arrow) had (E) a low signal level in T1-weighted imaging and (F) a high signal level in T2-weighted imaging. (G) In gadolinium-enhanced MRI of the arterial phase, the lesion was clearly enhanced. The SI of the nodule was comparable to that of the spleen. (H) In MRI at the splenic hilum level, a triangular accessory spleen (arrow) was visible with a degree of enhancement comparable to that of the orthotopic spleen. CT, computed tomography; MRI, magnetic resonance imaging; IPAS, intrapancreatic accessory spleen.

pancreas in each case. Lesions were located in the rear of the pancreatic tail with a straight edge or backward bulging in 5 cases (Fig. 1), occupied the middle of the pancreatic tail with mild forward and backward bulging in 2 cases (Fig. 2), and located in the forepart of the pancreatic tail and projected forward prominently in 1 case (Fig. 3). The shape of the lesions was round (n=3; Fig. 1), oval (n=4; Fig. 2) or triangular $(n=1$; Fig. 3). The largest diameter of the lesions was $2.3 \pm 1.0 \mathrm{~cm}$ (range, 1.3-3.7 cm) and the shortest was $1.4 \pm 0.6 \mathrm{~cm}$ (range, $0.9-2.4 \mathrm{~cm})$

Lesion density, signal and enhancement features. Unenhanced CT images indicated that the lesions in the 8 patients with IPAS were solid nodules with homogeneous density, equal to that of the main spleen. In comparison with pancreatic parenchyma, the lesions in 6 patients had slightly higher attenuation (Figs. 1A and 2A) and the lesion in 1 patient had slightly lower attenuation (Fig. 3A). However, in 1 patient (case 1; Fig. 4) the lesion had a similar attenuation level to the main spleen and was difficult to detect (Fig. 4A).

In the arterial phase, heterogeneous enhancement of the lesion was shown in 3 patients and 'zebra-patterned' enhancement was exhibited in 5 patients; in the portal venous phase, heterogeneous enhancement was shown in all 8 patients; and in the delayed phase, the degree of enhancement was reduced (Figs. 1, 4 and 5). The characteristic enhancement pattern of the lesions in these 8 patients was similar to that of the orthotopic spleen. In comparison with the pancreatic parenchyma, the IPAS of 1 patient exhibited a similar attenuation level (Fig. 4B and C) and the other 7 IPAS lesions had a higher attenuation than the surrounding pancreas in the three phases (Figs. 1-3 and 5).

In the MRI images, the IPAS for 7 patients exhibited low intensity signals in T1WI with and without fat suppression, but high intensity signals in T2WI compared with the surrounding pancreatic parenchyma, and the SI of the IPAS was comparable to that of the orthotopic spleen (Figs. 1 and 4-6).

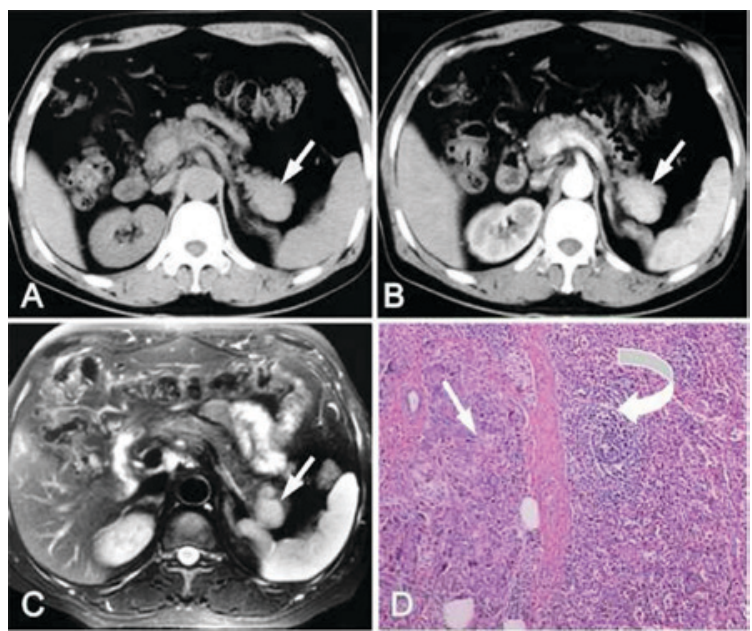

Figure 2. Case 2. IPAS was detected in a 44-year-old male due to upper abdominal pain and melena. (A) Unenhanced CT exhibited an oval, well-defined nodule bulging to the anterior and posterior of the pancreatic tail. (B) In a CT image of the arterial phase, homogeneous enhancement was observed for the lesion (arrow). (C) The lesion (arrow) had a high signal level in T2-weighted imaging. (D) The splenic tissue (curved arrow) located at the pancreatic parenchyma (arrow) was confirmed using histopathologic analysis (hematoxylin and eosin staining), and the IPAS was composed of red and white pulp, like normal spleen. Magnification, x100. IPAS, intrapancreatic accessory spleen; CT, computed tomography.

In the arterial phase, heterogeneous enhancement of the lesion was observed for 2 patients and 'zebra-patterned' enhancement was observed for 5 patients. In the portal venous phase, heterogeneous enhancement was shown for 7 patients, whereas in the delayed phase, the degree of enhancement was reduced. These results are similar to those obtained by CT. In comparison with the pancreas, the IPAS in 1 patient exhibited a similar degree of enhancement to the pancreas in the 3 phases, whereas the SI of the other 6 IPAS cases was higher than the pancreas in the 3 phases (Figs. 1G and 6C).

On DWI examination, the IPAS lesions in 5 patients presented high intensity signals when b-values of 0,100 and 


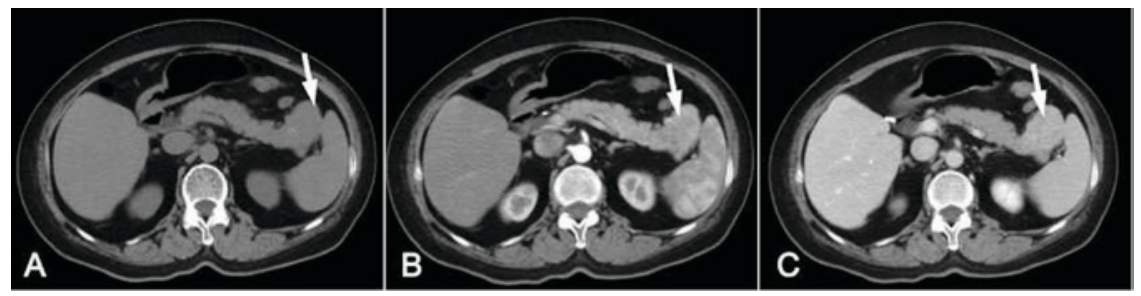

Figure 3. Case 7. A 63-year-old female was diagnosed with IPAS during a health examination. (A) Unenhanced computed tomography imaging exhibited a triangular lesion with low density in the forepart of the pancreatic tail, and the lesion was visible in front of the pancreatic tail (arrow). (B) The heterogeneous zebra-striped enhancement pattern within the lesion (arrow) was similar to the orthotopic spleen in the arterial phase. (C) The IPAS enhancement (arrow) tended to be homogeneous in the portal venous phase, and the density difference between the IPAS and its surrounding pancreatic tissue was evident. IPAS intrapancreatic accessory spleen.

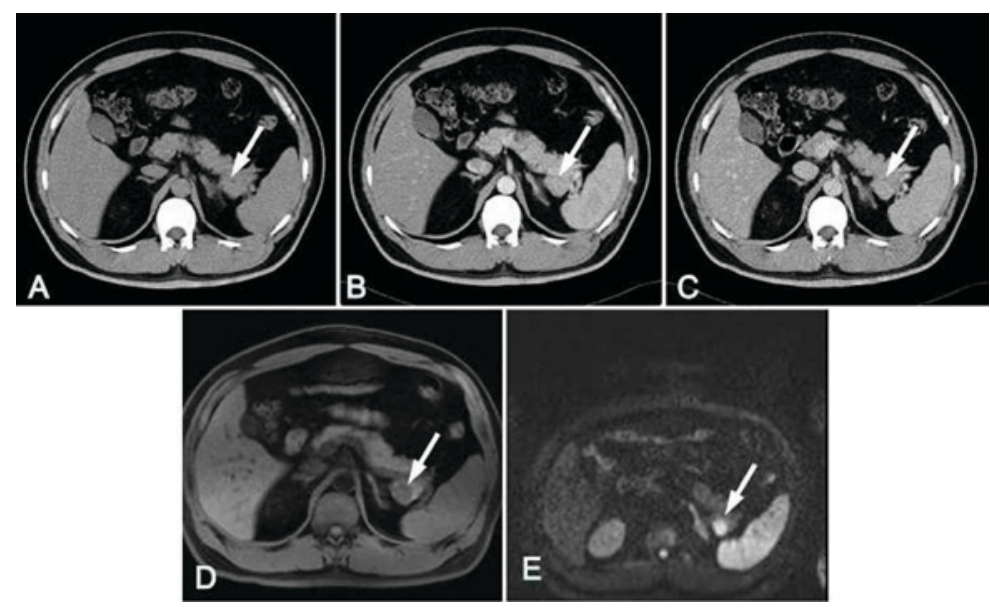

Figure 4. Case 1. A 56-year-old male was confirmed to have IPAS by surgical pathology. (A) The unenhanced CT image exhibited only slight enlargement of the pancreatic tail. In the (B) arterial phase and (C) delayed phase, similar attenuation levels were observed for the IPAS (arrow) and the surrounding pancreatic tissue. (D) In T1-weighted imaging, the IPAS exhibited a low signal in the pancreatic tissues (arrow). (E) In diffusion-weighted imaging using a high b-value $\left(600 \mathrm{sec} / \mathrm{mm}^{2}\right)$, the lesion (arrow) clearly had higher signal intensity than the pancreas. IPAS, intrapancreaticaccessory spleen; CT, computed tomography.

$600 \mathrm{sec} / \mathrm{mm}^{2}$ were used. When the b-value was $600 \mathrm{sec} / \mathrm{mm}^{2}$, the lesions clearly had higher SI and lower ADC values compared with the pancreas, and similar SI and ADC values to the orthotopic spleen (Figs. 4E and 6D). In these patients, the ADC value of the IPAS was $0.868 \pm 0.046 \mathrm{~mm}^{2} / \mathrm{sec}$, and the $\mathrm{ADC}$ value of the orthotopic spleen was $0.870 \pm 0.045 \mathrm{~mm}^{2} / \mathrm{sec}$; the difference between these two values was not significantly different $(\mathrm{t}=0.620, \mathrm{P}=0.587)$. The ADC value of the pancreatic tissue was $1.404 \pm 0.081 \times 10^{-3} \mathrm{~mm}^{2} / \mathrm{sec}$, which was significantly higher compared with that of the IPAS ( $\mathrm{P}<0.01$; Table II).

Accompanying presentations. In 2 of the 8 patients with IPAS, liver cirrhosis and splenomegaly complications were present, and in 1 of these 2 patients, hepatocellular carcinoma was confirmed by hepatic arteriography. The tumor exhibited a 'quick wash-in and wash-out' feature in dynamic enhanced CT imaging (Fig. 5). Accessory spleens were identified in 2 patients, and these accessory spleens had similar imaging features to those of the IPAS and orthotopic spleen (Fig. 1H).

Imaging findings of pancreatic splenosis. Splenosis in the pancreatic tail was observed in 1 patient who had undergone splenectomy. On the unenhanced CT image (CT value, $65 \mathrm{HU})$, the lesion exhibited high density and had a largest cross-sectional diameter of $2.2 \times 1.2 \mathrm{~cm}$. In comparison with the normal pancreas, the lesion appeared to have a slightly higher
SI in T1WI, and a lower SI in T2WI and DWI when a b-value of $600 \mathrm{sec} / \mathrm{mm}^{2}$ was used. Dynamic contrast-enhanced CT and MRI images demonstrated that the enhancement pattern of splenosis was similar to that of the normal spleen. In this case, several splenic nodules scattered in the upper left abdominal cavity were also detected with similar density, signals and enhancement patterns to those of the tail lesion (Fig. 7).

Pathological findings. The initial pathological diagnosis for 3 of the 9 patients was pancreatic tail tumor. Thus, resections of the distal pancreas and spleen were performed. In each case, the pancreatic tail specimen contained a round, smooth, dark-red nodule with a clear boundary. Cross-sectioning of the IPAS specimen revealed a reddish nodule surrounded by a fibrotic capsule, which separated it from the adjacent yellowish pancreatic tissue. Histological analysis revealed that the IPAS was composed of red and white pulp, which was similar to that of the normal spleen. The red pulp comprised numerous vascular sinuses. The lymphoid follicles and cells of the reticuloendothelial system lay between these sinuses, which constituted the white pulp (Fig. 2D).

\section{Discussion}

There have been few reports of splenosis in solid organs, and splenosis occurs most frequently in the liver according to these 


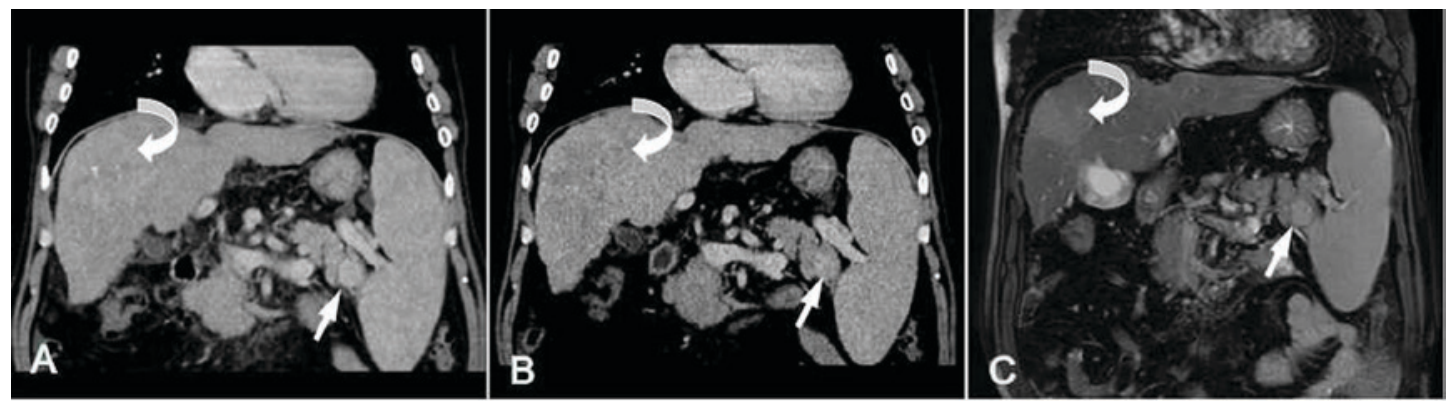

Figure 5. Case 3. CT and MRI images of a 57-year-old male with IPAS complicated with advanced cirrhosis and hepatocellular carcinoma. Coronal reconstructed CT images obtained in the (A) arterial phase and (B) delayed phase demonstrated that the density of the nodule (arrows) matched that of the orthotopic spleen, and was higher than that of the surrounding pancreatic tissue. In addition, a mass was observed in segment VII of the liver with an enhancement characteristic of 'quick wash in and wash out' (curved arrows). (C) Coronal MRI using true fast imaging with steady state precession revealed that the signal intensity of the nodule (arrow) was consistent with that of the orthotopic spleen, and the liver tumor (curved arrow) had slightly higher SI. CT, computed tomography; MRI, magnetic resonance imaging; IPAS, intrapancreatic accessory spleen.

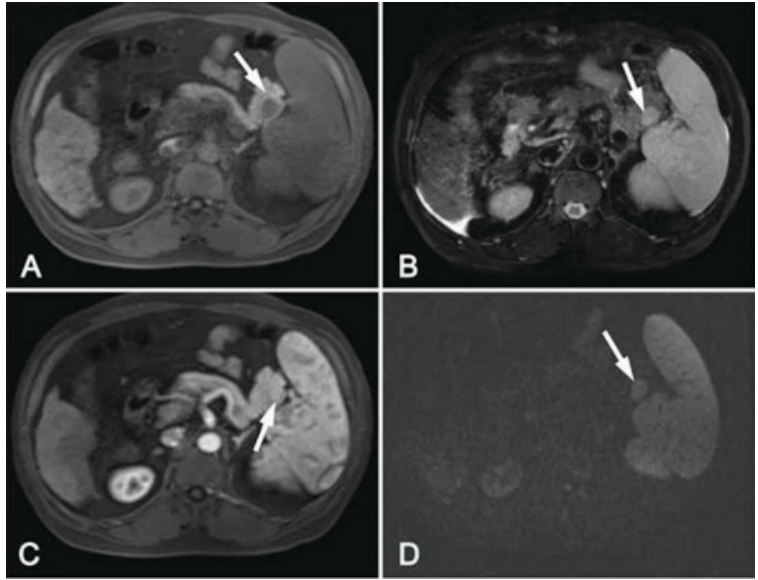

Figure 6. Case 3. The nodule in the pancreatic tail (arrows) had (A) a low signal level in T1-weighted imaging and (B) a high signal level in T2-weighted imaging. (C) Enhanced MRI presented a zebra-patterned enhancement in the arterial phase. (D) In diffusion-weighted imaging at a high b-value $\left(600 \mathrm{sec} / \mathrm{mm}^{2}\right)$, the lesion clearly had higher signal intensity than the surrounding pancreas. In these MRI sequences, the signals for the IPAS lesion were similar to those of the orthotopic spleen. MRI, magnetic resonance imaging; IPAS, intrapancreatic accessory spleen.

reports $(17,18)$. These cases have often been misdiagnosed as primary liver cancer and thus been treated using surgical resection $(17,18)$. Fiamingo et al (19) presented the first case of laparoscopic resection of pancreatic splenosis, in which the patient had a history of splenectomy. In that study, the clinical history and imaging findings of 2 patients with splenosis of the pancreatic tail were similar to the single patient with splenosis in the present study. It may be speculated that splenic tissue was brought into the pancreatic tail by splenectomy. Generally, the imaging features of intrapancreaticsplenosis are consistent with those of normal spleen. However, the case in the present study exhibited high density on pre-contrast CT images, slightly higher SI in T1WI and lower SI in T2WI and DWI, which was inconsistent with normal spleen. It is hypothesized that these unusual features might be associated with hemosiderosis caused by cirrhosis.

IPAS is not a rare condition; in an autopsy study of 2,700 patients, 61 of $364(17 \%)$ cases of accessory spleen in the pancreatic tail were detected (3). The tail of the pancreas was the second most common site of accessory splenunculi $(16-20 \%)(3,4,20,21)$, which was diagnosed by pathological analysis following surgery (22).

Prior to the regular use of CT and MRI scanning, IPAS was difficult to detect as symptoms are seldom and the consequences are not clinically significant (7). However, since abdominal multi-slice computed tomographic (MSCT) scans are now widely used in healthcare examinations and systemic surveys, IPAS cases have become easier to detect.

In the absence of secondary lesions, ectopic spleen in the pancreas usually does not require any aggressive treatment, unlike endocrine tumor and pancreatic adenocarcinoma $(5,9,15)$. Therefore, accurate diagnosis using preoperative imaging tools is important. However, comprehensive radiologic data on IPAS or pancreatic splenosis is lacking, and progressive investigation is urgently required. Thus, the present study describes the radiological features of 9 patients with intrapancreatic spleen and reviews other cases described in previous reports.

In the current study, the IPAS was observed to be a round or oval, smooth nodule with clear boundary surrounded by pancreatic tissue and a diameter of $1.3-3.7 \mathrm{~cm}$. These characteristics were consistent with those reported in previously studies $(2,11,23)$.

The density and SI of the intrapancreatic spleen were consistent with those of the orthotopic spleen in the majority of cases (8/9 cases). In comparison with the pancreatic parenchyma, the attenuation of the intrapancreatic spleen in the CT images was generally higher than that of the pancreas. The SI of the intrapancreatic spleen was low in T1WI and high in T2WI and DWI. Notably, high $b$-value $\left(b=600 \mathrm{sec} / \mathrm{mm}^{2}\right)$ DWI images provided greater soft tissue contrast than did T2WI with regard to SI. Thus, it offered superior diagnostic data for the depiction and characterization of the intrapancreatic spleen, even with inferior spatial resolution. The density and SI of the intrapancreatic spleen may be affected by pathological changes of the spleen, such as hemosiderosis, hypersplenismleukemia and lymphoma. For example, in 1 case in the current study, the CT density and MRI SI of the intrapancreatic spleen were inconsistent with those of normal spleen tissue, which was considered to be a result of substantial deposition of hemosiderin due to cirrhosis.

In the majority of cases, the enhancement patterns of the intrapancreatic spleen were consistent with those of the 
Table II. DWI signal strength and ADC values for the IPAS, pancreatic tissue and orthotopic spleen.

\begin{tabular}{lccc}
\hline Variable & IPAS & Pancreatic tissue & Orthotopic spleen \\
\hline $\begin{array}{l}\text { DWI signal strength } \\
\text { ADC value }\left(x 10^{-3} \mathrm{sec} / \mathrm{mm}^{2}\right)\end{array}$ & High & Low & High \\
& $0.868 \pm 0.046$ & $1.404 \pm 0.081^{\mathrm{a}}$ & $0.870 \pm 0.045$
\end{tabular}

${ }^{\mathrm{a}} \mathrm{P}<0.05$ vs. IPAS. DWI, diffusion-weighted imaging; IPAS, intrapancreatic accessory spleen; ADC, apparent diffusion coefficient.

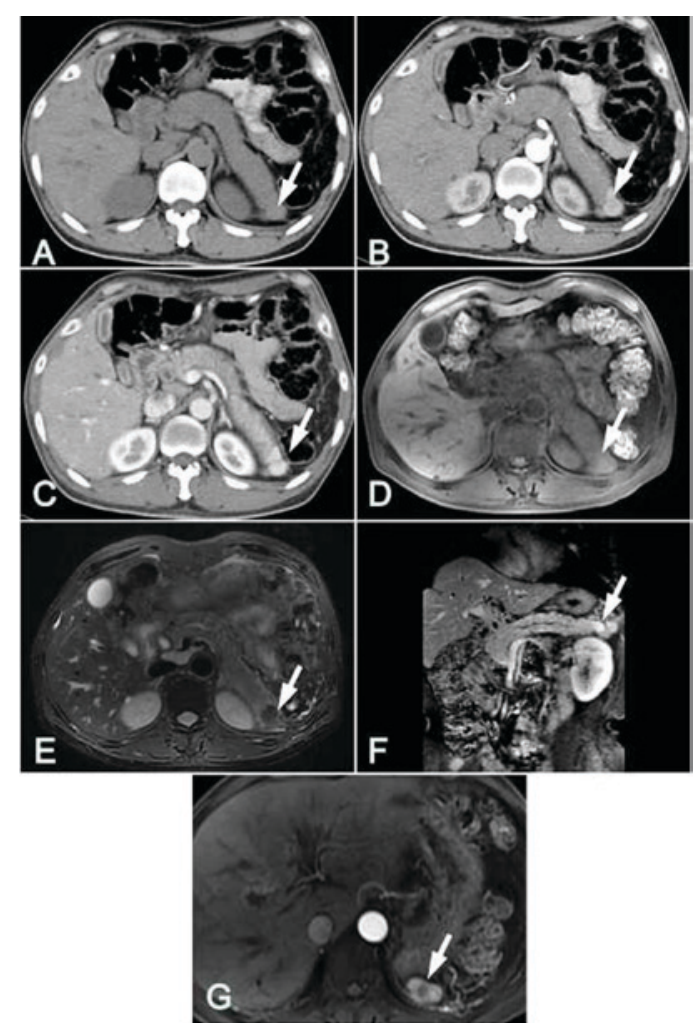

Figure 7. Case 4. Splenosis located in the pancreatic tail and intraperitoneal region of a 55-year-old man who had undergone splenectomy due to liver cirrhosis. (A) In a pre-contrast axial CT scan, the lesion (arrow) exhibited hyper-attenuation compared with the pancreas, and the CT value was $65 \mathrm{HU}$. Axial CT images were obtained in the (B) arterial phase and (C) portal venous phase. The lesion (arrow) exhibited higher attenuation than the surrounding pancreas in the two phases. (B) The inhomogeneous enhancement within the lesion (arrow) was similar to the zebra-striped enhancement of the spleen in the arterial phase. The lesion (arrow) had (D) a slightly higher signal level compared with the pancreas in T1-weighted imaging and (E) lower signal level in T2-weighted imaging. (F) On a consistent projection reconstructionimage obtained in the portal phase, the lesion (arrow) presented hyper-intensity compared with the pancreas. (G) In MRI of the base of the stomach, another splenosis was detected in the left subphrenic space (arrow), which had the same enhanced pattern as the intrapancreaticsplenosis. CT, computed tomography.

orthotopic or normal spleen and the enhancement degree was higher than that of the pancreas in three dynamic phases, which was consistent with the findings of Kim et al (2). The heterogeneous or zebra-patterned enhancement features, which are caused by different rates of flow through the cords of the red and white pulp in the arterial phase, may assist in diagnosis (24). Inhomogeneous enhancement patterns may be detected more frequently, even in small IPAS, by the application of MSCT, fast MRI sequences and high-power contrast agent delivery techniques, which enable the differentiation of IPAS from other hypervascular pancreatic tumors (2). However, Park et al (25) reported a case of ectopic spleen with different CT and MRI enhancement patterns from those of normal spleen, which was misdiagnosed as pancreatic tumor and then surgically resected. The attenuation and SI of IPAS may be lower than those of the pancreas in the arterial and pancreatic phases when splenic enhancement is retarded, such as in patients with liver cirrhosis (26). Therefore, for suspected cases with atypical dynamic enhancement patterns, DWI scanning is potentially useful to distinguish the lesion.

Nuclear medicine, such as ${ }^{99 \mathrm{~m}} \mathrm{Tc}-$ sulfur colloid and ${ }^{99 \mathrm{~m}} \mathrm{Tc}$ heat-damaged red blood cell scintigraphy, can be utilized as a confirmatory modality when IPAS or splenosis is suspected. It has been reported that pancreatic ectopic spleen can specifically take up ${ }^{99 m}$ Tc-heat-damaged red blood cells or sulfur colloid at a normal spleen tissue dose, which is helpful for the detection and definitive diagnosis of ectopic splenic tissue (14-16).

A small proportion of cases of intrapancreatic spleen may occur secondary to other diseases, including epidermoid cyst and inflammatory pseudotumor, have been detected in intrapancreatic spleens on rare occasions (27-29). The diagnosis of intrapancreatic spleen is challenging when secondary lesions are present.

In the present study, 3 cases were complicated with liver cirrhosis, and 1 of these 3 patients had secondary hepatocellular carcinoma. The detection of intrapancreatic spleen appeared to be more likely in patients with cirrhosis due to repeated imaging follow-up. Intrapancreatic spleen has often been misdiagnosed as pancreatic tumor causing patients to undergo unnecessary surgery $(5,12,15,19,25)$. Thus, it is recommended that the diagnosis of intrapancreatic spleen is included in the differential diagnosis of pancreatic hypervascular masses, such as pancreatic neuroendocrine tumors, solid pseudopapillarytumors and hypervascular metastases.

Neuroendocrine tumors, such as islet cell tumors, are often observed as small, round and hypervascular features in the arterial phase of CT and MRI images (9). However, nonfunctional neuroendocrine tumors are typically large and secondary to cystic necrosis, while IPAS usually presents as a small and homogeneous nodule. Unlike IPAS, islet cell tumors do not have a characteristic enhancement pattern (30). In DWI conducted using a high b-value, some endocrine neoplasms may present high SI and low ADC values (31), whereas IPAS exhibit homogeneous high signals in DWI.

Solid pseudopapillarytumor of the pancreas predominantly occurs in females aged 20-40 years according to a previous study by the present research team (32). In CT and MRI 
images, the lesions typically present as well-defined round shaped masses with a diameter $>5 \mathrm{~cm}$. In non-enhanced CT or MRI, the lesions exhibit heterogeneous density or SI. The solid parts of the tumor often present mild peripheral heterogeneous enhancement, and the degree of enhancement is usually lower than that of the pancreatic parenchyma.

Primary malignancies, such as lung cancer, breast cancer, and gastrointestinal and renal tumors, frequently metastasize to the pancreas. $\mathrm{Ng}$ et al (33) reported that renal cell carcinoma was the most common malignancy metastasizing to the pancreas, where the metastasis was solitary or multiple, well-defined and progressively enlarged. Pancreatic metastases of renal carcinoma were observed to exhibit rapid enhancement in the arterial phase, but were not easy to identify in the portal phase and were even less visible in the 120 -sec delayed phase. Although it was difficult to distinguish hypervascular metastasis from intrapancreatic spleen, a clinical history of known malignancy helped in the diagnosis (33).

In conclusion, a diagnosis of IPAS should be considered when a solidary lesion in the pancreatic tail has similar characteristics to the orthotopic spleen in pre-contrast and post-contrast enhanced CT and MRI images. Clearly elevated SI consistent with that of the spleen in DWI conducted using a high b-value is suggestive of a diagnosis of IPAS, and helps in the differential diagnosis. For intrapancreaticsplenosis, in addition to the above imaging features, a history of spleen trauma or splenectomy is crucial to the diagnosis.

\section{Acknowledgements}

Not applicable.

\section{Funding}

The present study was funded by the Natural Science foundation of Zhejiang Province (grant no. Y13H070008), the Medicine and Health Science and Technology Project of Zhejiang Province (grant nos. 2013KYA182 and 2012KYB176) and the Natural Science Foundation of Ningbo (grant nos. 2017A610146 and 2010A610052).

\section{Availability of data and materials}

The datasets used and/or analyzed during the current study are available from the corresponding author on reasonable request.

\section{Authors' contributions}

QD and ZR collected the cases, analysed the imaging features and drafted the paper. JW designed the study, analyzed the data and revised the paper. XM and JZ collected the cases and analysed imaging features. GS and HG collected the cases and analysed the data. $\mathrm{CZ}$ revised the paper. $\mathrm{HJ}$ performed the pathological examinations.

\section{Ethics approval and consent to participate}

The present retrospective review of patient data was approved by the Ethics Committee of Ningbo University Hospital.

\section{Consent for publication}

Not applicable.

\section{Competing interests}

The authors declare that they have no competing interests.

\section{References}

1. Movitz D: Accessory spleens and experimental splenosis. Principles of growth. Chic Med Sch Q 26: 183-187, 1967.

2. Kim SH, Lee JM, Han JK, Lee JY, Kim KW, Cho KC and Choi BI: Intrapancreatic accessory spleen: Findings on MR Imaging, CT, US and scintigraphy, and the pathologic analysis. Korean J Radiol 9: 162-174, 2008.

3. Halpert B and Alden ZA: Accessory spleens in or at the tail of the pancreas. A survey of 2700 additoonal necropsies. Arch Pathol 77: 652-654, 1964.

4. Halpert B and Gyorkey F: Lesions observed in accessory spleens of 311 patients. Am J Clin Pathol 32: 165-168, 1959.

5. Matthaei H, Schmelzle M, Braunstein S, Bölke E and Peiper M: Pancreatic incidentalomas: A growing clinical challenge exemplified by an intrapancreatic accessory spleen. Wien Klin Wochenschr 123: 186-188, 2011.

6. Churei $\mathrm{H}$, Inoue $\mathrm{H}$ and Nakajo $\mathrm{M}$ : Intrapancreatic accessory spleen: Case report. Abdom Imaging 23: 191-193, 1998.

7. Sica GT and Reed MF: Case 27: Intrapancreatic accessory spleen. Radiology 217: 134-137, 2000.

8. Tozbikian G, Bloomston M, Stevens R, Ellison EC and Frankel WL: Accessory spleen presenting as a mass in the tail of the pancreas. Ann Diagn Pathol 11: 277-281, 2007.

9. Uchiyama S, Chijiiwa K, Hiyoshi M, Ohuchida J, Imamura N, Nagano M, Hidaka H, Yorita K, Akiyama Y and Nishiura M: Intrapancreatic accessory spleen mimicking endocrine tumor of the pancreas: Case report and review of the literature. J Gastrointest Surg 12: 1471-1473, 2008.

10. Spencer LA, Spizarny DL and Williams TR: Imaging features of intrapancreatic accessory spleen. Br J Radiol 83: 668-673, 2010.

11. Low G, Panu A, Millo N and Leen E: Multimodality imaging of neoplastic and nonneoplastic solid lesions of the pancreas. Radiographics 31: 993-1015, 2011.

12. Kawamoto S, Johnson PT, Hall H, Cameron JL, Hruban RH and Fishman EK: Intrapancreatic accessory spleen: CT appearance and differential diagnosis. Abdom Imaging 37: 812-827, 2012.

13. Dodds WJ, Taylor AJ, Erickson SJ, Stewart ET and Lawson TL: Radiologic imaging of splenic anomalies. American Journal of Roentgenology 155: 805-810, 2013.

14. Ota T, Tei M, Yoshioka A, Mizuno M, Watanabe S, Seki M, Nakata H, Yamamoto I and Morita R: Intrapancreatic accessory spleen diagnosed by technetium-99m heat-damaged red blood cell SPECT. J Nucl Med 38: 494-495, 1997.

15. Brasca LE, Zanello A, De Gaspari A, De Cobelli F, Zerbi A, Fazio F and Del Maschio A: Intrapancreatic accessory spleen mimicking a neuroendocrine tumor: Magnetic resonance findings and possible diagnostic role of different nuclear medicine tests. Eur Radiol 14: 1322-1323, 2004.

16. Belkhir SM, Archambaud F, Prigent A and Chaumet-Riffaud P: Intrapancreatic accessory spleen diagnosed on radionuclide imaging. Clin Nucl Med 34: 642-644, 2009.

17. Kim KA, Park CM, Kim CH, Choi SY, Park SW, Kang EY, Seol HY and Cha IH: An interesting hepatic mass: Splenosis mimicking a hepatocellular carcinoma (2003:9b). Eur Radiol 13: 2713-2715, 2003

18. Abu Hilal M, Harb A, Zeidan B, Steadman B, Primrose JN and Pearce NW: Hepatic splenosis mimicking HCC in a patient with hepatitis $\mathrm{C}$ liver cirrhosis and mildly raised alpha feto protein; the important role of explorative laparoscopy. World J Surg Oncol 7: 1, 2009.

19. Fiamingo P, Veroux M, Da Rold A, Guerriero S, Pariset S, Buffone A and Tedeschi U: A rare diagnosis for a pancreatic mass: Splenosis. J Gastrointest Surg 8: 915-916, 2004.

20. Varga I, Galfiova P, Adamkov M, Danisovic L, Polak S, Kubikova E and Galbavy S: Congenital anomalies of the spleen from an embryological point of view. Med Sci Monit 15: RA269-RA276, 2009 
21. Mortelé KJ, Mortelé B and Silverman SG: CT features of the accessory spleen. AJR Am J Roentgenol 183: 1653-1657, 2004.

22. Hwang HS, Lee SS, Kim SC, Seo DW and Kim J: Intrapancreatic accessory spleen: Clinicopathologic analysis of 12 cases. Pancreas 40: 956-965, 2011.

23. Kim SH, Lee JM, Han JK, Lee JY, Kang WJ, Jang JY, Shin KS, Cho KC and Choi BI: MDCT and superparamagnetic iron oxide (SPIO)-enhanced MR findings of intrapancreatic accessory spleen in seven patients. Eur Radiol 16: 1887-1897, 2006.

24. Paterson A, Frush DP, Donnelly LF, Foss JN, O'Hara SM and Bisset GS 3rd: A pattern-oriented approach to splenic imaging in infants and children. Radiographics 19: 1465-1485, 1999.

25. Park JS, Kim WJ, Jeong YG, Park YS, Koo HC, Lee TI, Choi GC and Kim S: A case of intrapancreatic accessory spleen mistaken as a pancreatic mass due to different enhancing pattern from normal spleen. Korean J Gastroenterol 58: 357-360, 2011.

26. Blomley MJ, Kormano M, Coulden R, Lim-Dunham J, Dawson P and Lipton MJ: Splenic blood flow: Evaluation with computed tomography. Acad Radiol 4: 13-20, 1997.

27. Davidson ED, Campbell WG and Hersh T: Epidermoid splenic cyst occurring in an intrapancreatic accessory spleen. Dig Dis Sci 25: 964-917, 1980

28. Hu S, Zhu L, Song Q and Chen K: Epidermoid cyst in intrapancreatic accessory spleen: Computed tomography findings and clinical manifestation. Abdom Imaging 37: 828-833, 2012.
29. Okura N, Mori K, Morishita Y, Oda T, Tanoi T and Minami M: Inflammatory pseudotumor of the intrapancreatic accessory spleen: Computed tomography and magnetic resonance imaging findings. Jpn J Radiol 30: 171-175, 2012.

30. Sukaiti R, Robinson K and Menias C: Retrospective review of cross sectional imaging findings of pancreatic non-functional islet cell tumor (NFICT) and its hepatic metastases. Oman Med J 26: 39-42, 2011

31. Kurata Y, Kido A, Moribata Y, Kameyama K, Himoto Y, Minamiguchi S, Konishi I and Togashi K: Diagnostic performance of MR imaging findings and quantitative values in the differentiation of seromucinous borderline tumour from endometriosis-related malignant ovarian tumour. Eur Radiol 27: 1695-1703, 2017

32. MaXL, Wang JH, Jiang H, Lu JP and Liu Q: Solid-pseudopapillary tumor of pancreas: Different types of imaging features and their correlation with pathological findings. Zhonghua Yi Xue Za Zhi 92: 170-174, 2012 (In Chinese).

33. Ng CS, Loyer EM, Iyer RB, David CL, DuBrow RA and Charnsangavej C: Metastases to the pancreas from renal cell carcinoma: Findings on three-phase contrast-enhanced helical CT. AJR Am J Roentgenol 172: 1555-1559, 1999. 